\title{
Multiple coverings with closed polygons
}

\author{
István Kovács* \\ Budapest University of Technology and Economics \\ Budapest, Hungary \\ kovika91@gmail.com \\ Géza Tóth ${ }^{\dagger}$ \\ Alfréd Rényi Institute of Mathematics \\ Budapest, Hungary \\ geza@renyi.hu
}

Submitted: Mar 26, 2014; Accepted: Jan 15, 2015; Published: Jan 27, 2015

Mathematics Subject Classifications: 52C15

\begin{abstract}
A planar set $P$ is said to be cover-decomposable if there is a constant $k=k(P)$ such that every $k$-fold covering of the plane with translates of $P$ can be decomposed into two coverings. It is known that open convex polygons are cover-decomposable. Here we show that closed, centrally symmetric convex polygons are also coverdecomposable. We also show that an infinite-fold covering of the plane with translates of $P$ can be decomposed into two infinite-fold coverings. Both results hold for coverings of any subset of the plane.
\end{abstract}

Keywords: multiple covering, decomposition

\section{Introduction}

The study of multiple coverings was initiated by Davenport and L. Fejes Tóth about 60 years ago [1]. Let $\mathcal{S}=\left\{S_{i} \mid i \in I\right\}$ be a collection of sets in the plane. We say that $\mathcal{S}$ is an $m$-fold covering if every point of the plane is contained in at least $m$ elements of $\mathcal{S}$. A 1-fold covering is simply called a covering. Clearly, the union of $k$ coverings is always a $k$-fold covering, but it is easy to see that the converse is not necessarily true, not even in the special case when $\mathcal{S}$ is a collection of translates of a given set.

\footnotetext{
*Supported by Támop - 4.2.2.B-10/1-2010-0009 and by Supported by Hungarian Science Foundation EuroGIGA Grant OTKA NN 102029.

†Supported by Hungarian Science Foundation Grants OTKA K-83767 and NN-102029.
} 
Definition 1. A planar set $S$ is said to be cover-decomposable if there exists a (minimal) constant $k=k(S)$ such that every $k$-fold covering of the plane with translates of $S$ can be decomposed into two coverings.

J. Pach proposed the problem of determining all cover-decomposable sets in 1980 [4]. He conjectured that all planar convex sets are cover-decomposable. Today there is a vast literature on this subject, partly because of its theoretical interest [6], and partly because of its applications in the sensor cover problem in sensor network scheduling [3].

Pach verified his conjecture for centrally symmetric open convex polygons [5]. The next result in this direction was by G. Tardos and G. Tóth [11], they proved that open triangles are cover-decomposable. Finally, D. Pálvölgyi and G. Tóth [10] proved that all open convex polygons are cover-decomposable.

Observe, that all of these general positive results hold only for open sets. The reason is that - based on the ideas of Pach [5] - all proofs reduce the problem to a finite problem, and that reduction works only for open sets. We believe that in fact all these results can be generalized for the closed version. The main result of this paper is the first step in this direction.

Theorem 2. Every centrally symmetric closed convex polygon is cover-decomposable.

From the other direction, J. Pach, G. Tardos, and G. Tóth [7] proved that (open and closed) concave quadrilaterals are not cover-decomposable. It was generalized by D. Pálvölgyi [8] who showed for a large class of concave polygons that they are not coverdecomposable. It is still not known whether there exists a cover-decomposable concave polygon. Very recently, D. Pálvölgyi [9] refuted Pach's conjecture. He proved that open and closed sets with smooth boundary are not cover-decomposable. In particular, the unit disc is not cover-decomposable.

Splitting infinite-fold coverings can lead to very deep problems. Elekes, Mátrai and Soukup [2] constructed an infinite-fold covering of the line by translates of a closed set, whose decomposability is independent of ZFC. We believe that it is not the case for coverings of the plane with translates of a convex closed set.

It follows directly from Theorem 2 that an infinite-fold covering of the plane with translates of a closed, convex, centrally symmetric polygon is decomposable into two coverings. We prove the following stronger result.

Theorem 3. Let $S$ be a closed, convex, centrally symmetric polygon. Then every infinitefold covering of the plane with translates of $S$ can be decomposed into two infinite-fold coverings.

Cover-decomposability has many other versions, instead of the plane, we can investigate, and decompose coverings of an arbitrary subset of the plane. We can consider only coverings with finite, countably many, or arbitrarily many translates. In the last section we review some of these versions of cover-decomposability. Our Theorems 2 and 3 hold for each of these versions, with the same proof. 


\section{Centrally symmetric closed convex polygons; Proof of Thm. 2}

\subsection{Taking the dual, reduction to wedges}

Just like in most of the papers about cover-decomposability, we formulate and solve the problem in its dual form. The idea is originally due to J. Pach [5]. Suppose that $S$ is an open or closed, centrally symmetric convex polygon, its vertices are $v_{1}, v_{2}, \ldots, v_{2 n}$, ordered clockwise. Indices are understood modulo $2 n$.

Definition 4 . For any two points, $a$ and $b$, let $\overrightarrow{a b}$ denote the halfline whose endpoint is $a$ and goes through $b$. Let $\arg (\overrightarrow{a b})$ denote the counterclockwise angle from the positive $x$ axis to $\overrightarrow{a b}$.

Definition 5. For every $i, 1 \leqslant i \leqslant 2 n$, let $E_{i}$ denote the convex wedge whose bounding halflines are the translates of $\overrightarrow{v_{i} v_{i-1}}$ and $\overrightarrow{v_{i} v_{i+1}}$. If $S$ is closed (resp. open), then let $E_{i}$ also be closed (resp. open). $E_{i}$ is called the wedge that belongs to vertex $v_{i}$ of $S$. We say that a wedge $E$ belongs to $S$, or $E$ is an $S$-wedge, if it belongs to one of its vertices. For any point $p$, let $E_{i}(p)$ denote the translate of $E_{i}$ such that its apex is in $p$.

A planar point set is bounded, if it is contained in a disc. Now we can state the dual version of Theorem 2 .

Theorem 6. Let $S$ be a centrally symmetric closed convex polygon, with vertices $v_{1}, v_{2}$, $\ldots, v_{2 n}$, ordered clockwise. Then there is an $m=m(S)>0$ with the following property.

Any bounded point set $\mathcal{H}$ can be colored with red and blue such that for any translate of an $S$-wedge, $E_{i}(p)$, if $\left|E_{i}(p) \cap \mathcal{H}\right| \geqslant m$, then $E_{i}(p) \cap \mathcal{H}$ contains points of both colors.

\section{Proof of Theorem 2 from Theorem 6.}

Let $x=x(S)$ be a number with the property that a square of side $x$ intersects at most two consecutive sides of $S$. Divide the plane into squares of side $x$, by a square grid. (Let common vertical boundaries belong to the left squares, common horizontal boundaries belong to the lower squares.) There is a constant $k^{\prime}$ such that any translate of $S$ intersects at most $k^{\prime}$ little squares. For any point $p$, let $S(p)$ denote the translate of $S$ so that its center is at $p$. Let $\mathcal{H}=\left\{S_{i} \mid i \in I\right\}$ be a collection of translates of $S$ that form a $k=k^{\prime} m$-fold covering, where $m=m(S)$ from Theorem 6 . For every $i \in I$, let $c_{i}$ be the center of $S_{i}$. Let $\mathcal{H}^{\prime}=\left\{c_{i} \mid i \in I\right\}$ be the set of centers. For any point $a, a \in S_{i}$ if and only if $c_{i} \in S(a)$. Therefore, for every point $a, S(a)$ contains at least $k$ points of $\mathcal{H}^{\prime}$.

The collection $\mathcal{H}$ can be decomposed into two coverings if and only if the points of the set $\mathcal{H}^{\prime}$ can be colored with two colors, such that every translate of $S$ contains points of both colors.

Color the points of $\mathcal{H}^{\prime}$ in each square separately, satisfying the conditions of Theorem 6. Now return to the covering $\mathcal{H}$ and color each translate of $S$ in $\mathcal{H}$ to the color of its center. We claim that both the red and the blue translates form a covering. Let $p$ be an arbitrary point, we have to show that it is covered by a translate of both colors. Or equivalently, $S(p)$ contains points of $\mathcal{H}^{\prime}$ of both colors. Since $S(p)$ contains at least $k$ 
points of $\mathcal{H}^{\prime}$, it contains at least $k / k^{\prime}=m$ points in one of the little squares $Q$. But $S(p)$ intersects $Q$ "like a wedge" that is, $S(p) \cap Q=E(q) \cap Q$ for some $S$-wedge $E$ and point q. Therefore, by Theorem 6, $S(p) \cap Q$ contains points of $\mathcal{H}^{\prime}$ of both colors.

Now we "only" have to prove Theorem 6. We need some preparation.

\subsection{Some properties of boundary points}

Theorem 6 has been proved by J. Pach [5] in the special case when $\mathcal{H}$ is finite. Some parts of our proof are just modifications of his argument, but some other parts are completely new.

Let $S$ be a centrally symmetric, open or closed convex polygon, its vertices are $v_{1}, v_{2}, \ldots, v_{2 n}$, ordered clockwise, the $S$-wedges are $E_{1}, E_{2}, \ldots, E_{2 n}$, respectively. Indices are understood $\bmod 2 n$, e. g. $v_{2 n+1}=v_{1}, E_{2 n+1}=E_{1}$. Let $\mathcal{H}$ be a bounded point set.

Definition 7. If $S$ is closed (resp. open), a point $p \in \mathcal{H}$ is called an $E_{i}$-boundary point if $E_{i}(p) \cap \mathcal{H}=\{p\}$ (resp. $\left.E_{i}(p) \cap \mathcal{H}=\emptyset\right)$.

Let $\mathbb{B}_{i}=\mathbb{B}_{i}(\mathcal{H})$ denote the set of $E_{i}$-boundary points of $\mathcal{H}$. Let $\mathbb{B}=\mathbb{B}(\mathcal{H})=\cup_{i=1}^{\infty} \mathbb{B}_{i}$ denote the set of all boundary points of $\mathcal{H}$, it is called the boundary of $\mathcal{H}$. The other points of $\mathcal{H}$ are called interior points.

For every $i, 1 \leqslant i \leqslant 2 n$, we introduce an ordering of the $E_{i}$-boundary $\mathbb{B}_{i}$. These orders together will give a cyclic ordering of the boundary $\mathbb{B}$, where some boundary vertices may appear twice. Let $\ell_{i}$ be a line perpendicular to the angular bisector of $E_{i}$. Direct $\ell_{i}$ so that $E_{i}$ can be translated to the left side of $\overrightarrow{\ell_{i}}$. There is a natural ordering of the points of $\overrightarrow{\ell_{i}}$. For $x, y \in \overrightarrow{\ell_{i}}$ we say that $x$ precedes $y$ ( $y$ follows $x$ ) if the vector $\overrightarrow{x y}$ points to the same direction as $\overrightarrow{\ell_{i}}$. Orthogonally project the points of $\mathbb{B}_{i}$ to $\overrightarrow{\ell_{i}}$, the image of $p$ is $\pi(p)$.

It is easy to see that for $1 \leqslant i \leqslant 2 n$, the map $\pi_{i}$ is injective. If $p_{1}, p_{2} \in \mathbb{B}_{i}$ and $\pi_{i}\left(p_{1}\right)=\pi_{i}\left(p_{2}\right)$, then either $p_{2} \in E_{i}\left(p_{1}\right)$ or $p_{1} \in E_{i}\left(p_{2}\right)$, but both of them are impossible since both $p_{1}$ and $p_{2}$ are $E_{i}$-boundary points.

Definition 8 . Let $1 \leqslant i \leqslant 2 n$. For any two $E_{i}$-boundary points $p_{1}$ and $p_{2}$, let $p_{1} \prec_{i} p_{2}$ if and only if $\pi_{i}\left(p_{1}\right)$ precedes $\pi_{i}\left(p_{2}\right)$ on $\overrightarrow{\ell_{i}}$.

The relation $\pi_{i}$ is a linear ordering on $\mathbb{B}_{i}$. Based on $\pi_{i}$, we can define intervals on $\mathbb{B}_{i}$, for example,

$$
\left[p_{1}, p_{2}\right]=\left\{p \in \mathbb{B}_{i}: \pi_{i}(p) \in\left[\pi_{i}\left(p_{1}\right), \pi_{i}\left(p_{2}\right)\right]\right\} .
$$

We say that the first half of the interval $\left[p_{1}, p_{2}\right]$ is

$$
\left\{p \in \mathbb{B}_{i}: \pi_{i}(p) \in\left[\pi_{i}\left(p_{1}\right), \frac{\pi\left(p_{1}\right)+\pi_{i}\left(p_{2}\right)}{2}\right]\right\}
$$

We define the open interval $\left(p_{1}, p_{2}\right)$ similarly as $\left[p_{1}, p_{2}\right]$, and we define the second half of an interval similarly as the first half. 
Claim 9. Suppose that $p \in \mathbb{B}_{i}$ and $p \in \mathbb{B}_{i+1}$, that is, $p$ is a boundary point with respect to both $E_{i}$ and $E_{i+1}$. Let $\ell$ be the line through $p$, parallel to $v_{i} v_{i+1}$. Then one of the closed halfplanes defined by $\ell$ contains all points of $\mathcal{H}$.

Proof. If $p$ is a boundary point with respect to both $E_{i}$ and $E_{i+1}$, then $E_{i}(p) \cap \mathcal{H}=$ $E_{i+1}(p) \cap \mathcal{H}=\emptyset$ if $S$ is open, and $\{p\}$ if $S$ is closed. But since $S$ is convex and centrally symmetric, $E_{i}(p) \cup E_{i+1}(p)$ contains an open halfplane bounded by $\ell$. This halfplane does not contain any point of $\mathcal{H}$, therefore, its complement satisfies the conditions.

It is easy to see now that if $S$ is closed, then there is at most one point $p \in \mathcal{H}$ that is a boundary point with respect to both $E_{i}$ and $E_{i+1}$. If $S$ is open and both $p$ and $q$ are such boundary points, then $p \prec_{i} q$ if and only if $p \prec_{i+1} q$. It also follows from Claim 9 that if $p$ is a boundary point with respect to both $E_{i}$ and $E_{i+1}$ and $q$ is a boundary point with respect to $E_{i}$ but not $E_{i+1}$, then $p \prec_{i} q$.

There could be other types of boundary points with respect to more than one wedge.

Definition 10. A point $p \in \mathcal{H}$ is a singular boundary point if there are numbers $1 \leqslant$ $i_{1}<n_{1}<i_{2}<n_{2} \leqslant 2 n$, or $1 \leqslant n_{1}<i_{1}<n_{2}<i_{2} \leqslant 2 n$ such that $p$ is a boundary point with respect to $E_{i_{1}}$ and $E_{i_{2}}$, but not a boundary point with respect to $E_{n_{1}}$ and $E_{n_{2}}$, see in Figure 1. Non-singular boundary points are called regular boundary points.

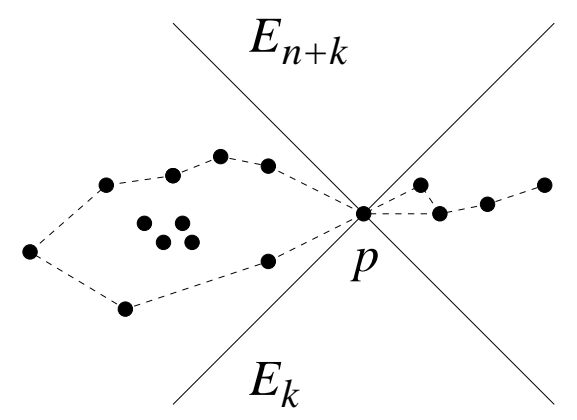

Figure 1: Point $p$ is a singular boundary point

This concept, just like the next two claims, are again basically from [5].

Claim 11. If $p$ is a singular $E_{i}$-boundary point, then it is a boundary point with respect to $E_{i}$ and $E_{i+n}$ (the reflection of $E_{i}$ ) and no other wedge.

Proof. Suppose that $1 \leqslant i_{1}<n_{1}<i_{2}<n_{2} \leqslant 2 n, p$ is a boundary point with respect to $E_{i_{1}}$ and $E_{i_{2}}$, but it is not a boundary point with respect to $E_{n_{1}}$ and $E_{n_{2}}$, and $i_{1}+n \neq i_{2}$. Assume without loss of generality that $i_{1}=1, i_{2}=k \leqslant n$. Then $E_{1}(p)$ and $E_{k}(p)$ do not contain any point of $\mathcal{H}$, different from $p$. It follows from the convexity of $S$, that $E_{n_{1}}(p) \subset$ $E_{1}(p) \cup E_{k}(p)$, therefore, $p$ is a boundary point with respect to $E_{n_{1}}$, a contradiction, see Figure 2. The argument is the same if we have $1 \leqslant n_{1}<i_{1}<n_{2}<i_{2} \leqslant 2 n$. 


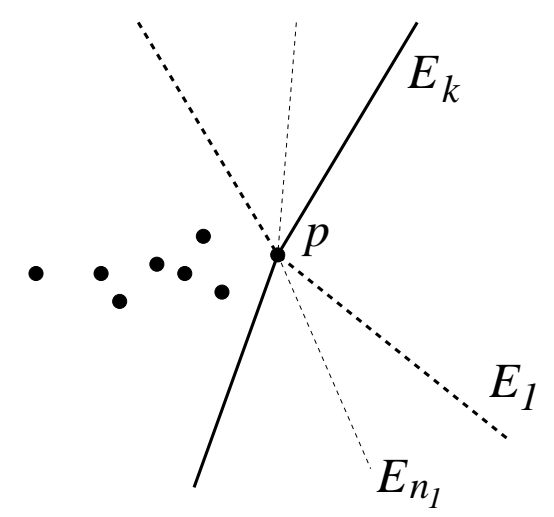

Figure 2: If $p$ is a boundary point with respect to $E_{1}$ and $E_{k}$, then it is also a boundary point with respect to $E_{n_{1}}$.

Now we show that all singular boundary points are of the "same type".

Claim 12. If $p$ is a singular boundary point with respect to $E_{i}$ and $E_{i+n}$, then there is no singular boundary point with respect to some other pair of wedges.

Proof. It Suppose that $p$ and $q$ are singular boundary points with respect to different pairs of wedges, say, $p$ with respect to $E_{1}$ and $E_{n+1}, q$ with respect to $E_{k}$ and $E_{n+k}, 1<k \leqslant n$. Suppose for simplicity, that $\arg \left(\overrightarrow{v_{1} v_{2}}\right)<\arg \left(\overrightarrow{v_{2 n} v_{1}}\right)$ and $\arg \left(\overrightarrow{v_{k} v_{k+1}}\right)<\arg \left(\overrightarrow{v_{k-1} v_{k}}\right)$. It follows that either

$$
\arg \left(\overrightarrow{v_{1} v_{2}}\right) \leqslant \arg (\overrightarrow{p q}) \leqslant \arg \left(\overrightarrow{v_{2 n} v_{1}}\right)
$$

or

$$
\arg \left(\overrightarrow{v_{1} v_{2}}\right) \leqslant \arg (\overrightarrow{q p}) \leqslant \arg \left(\overrightarrow{v_{2 n} v_{1}}\right) .
$$

Suppose without loss of generality that (1) holds. Since $q$ is a boundary point with respect to $E_{k}$ and $E_{n+k}$,

$$
\arg \left(\overrightarrow{v_{k} v_{k+1}}\right) \leqslant \arg (\overrightarrow{p q}) \leqslant \arg \left(\overrightarrow{v_{k-1} v_{k}}\right) .
$$

(Note, that if $S$ is closed then the above inequalities are strict inequalities.) But, because of the convexity of $S,(1)$ and (3) can hold simultaneously only if $k=2$ and

$$
\arg \left(\overrightarrow{v_{1} v_{2}}\right)=\arg (\overrightarrow{p q})
$$

But in this case, $q$ is also an $E_{1}$-boundary point, so it is not singular, a contradiction, see Figure 3.

From now on, suppose, without loss of generality, that all singular boundary points of $\mathcal{H}$ are $E_{1^{-}}$and $E_{n+1^{-}}$-boundary points. Observe, that if $p$ and $q$ are singular boundary points with respect to $E_{1}$ and $E_{n+1}$, then $p \prec_{1} q \Leftrightarrow q \prec_{n+1} p$. From now on, the type of a boundary point $p$ is the smallest $i$ such that $p$ is an $E_{i}$-boundary point.

In the set $\mathbb{B}$ of boundary points, substitute each singular boundary point $p$ by $p^{\prime}$ and $p^{\prime \prime}$, such that $p^{\prime}$ is an $E_{1}$-boundary point, $p^{\prime \prime}$ is an $E_{n+1}$-boundary point. Let $\mathbb{B}^{\prime}$ be the resulting set. For $p, q \in \mathbb{B}^{\prime}$, let $p \prec q$ if 


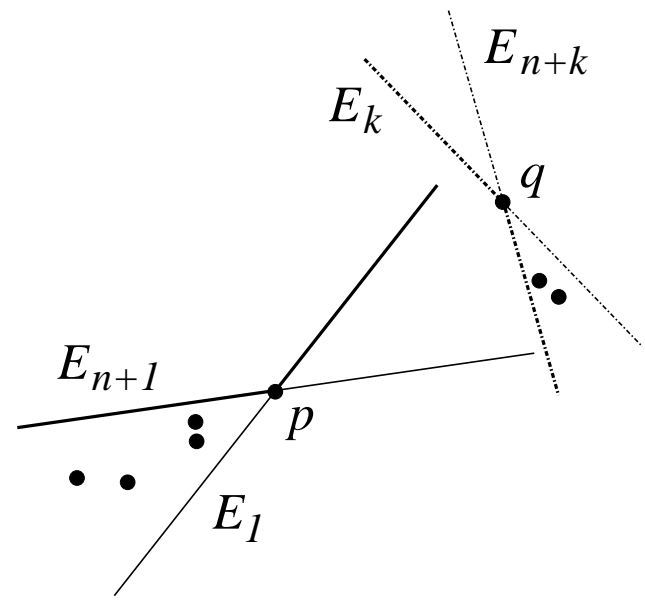

Figure 3: It is impossible that $p$ and $q$ are singular boundary points with respect to different pairs of opposite wedges.

- $p$ is of type $i, q$ is of type $j$, and $1 \leqslant i<j \leqslant 2 n$,

or

- both $p$ and $q$ are of type $i$, and $p \prec_{i} q$.

Relation $\prec$ gives a linear ordering on $\mathbb{B}^{\prime}$. We have the elements in the following order:

- Boundary points with respect to both $E_{2 n}$ and $E_{1}$, ordered according to $\prec_{2 n}$ and $\prec_{1}$;

- $E_{1}$-boundary points, ordered according to $\prec_{1}$;

- Boundary points with respect to both $E_{1}$ and $E_{2}$, ordered according to $\prec_{1}$ and $\prec_{2}$;

- $E_{2}$-boundary points, ordered according to $\prec_{2}$;

- Boundary points with respect to both $E_{2}$ and $E_{3}$, ordered according to $\prec_{2}$ and $\prec_{3}$;

- $E_{3}$-boundary points, ordered according to $\prec_{3}$;

- ...

- $E_{2 n}$-boundary points, ordered according to $\prec_{2 n}$.

If we project the points of $\mathbb{B}^{\prime}$ on a circle, then there is a natural way to define intervals on $\mathbb{B}^{\prime}$, and then also on $\mathbb{B}$. Now we define them precisely. 
Definition 13. An $I \subset \mathbb{B}^{\prime}$ subset is called an interval of $\mathbb{B}^{\prime}$, if one of the following two conditions hold.

(i) If $p \prec q \prec r$ and $p, r \in I$, then $q \in I$.

(ii) If $p \prec r, p, r \in I$, and either $q \prec p$ or $r \prec q$, then $q \in I$.

A subset $I \subset \mathbb{B}$ is called an interval of $\mathbb{B}$ if the corresponding subset $I^{\prime} \subset \mathbb{B}^{\prime}$ is an interval of $\mathbb{B}^{\prime}$.

An interval of $\mathbb{B}$ or $\mathbb{B}^{\prime}$ is called homogeneous if all its points are $E_{i}$-boundary points, for some $i$.

Claim 14. A translate of an $S$-wedge $E_{l}$ intersects $\mathbb{B}$ in at most two intervals.

Proof. Consider a translate of an $S$-wedge, say, $E_{l}(z), 1 \leqslant l \leqslant n$. Assume for simplicity that $\arg \left(\overrightarrow{v_{l} v_{l-1}}\right)<\arg \left(\overrightarrow{v_{l} v_{l+1}}\right)$. Suppose that $p$ is an $E_{i}$-boundary point, $q$ is an $E_{j^{-}}$ boundary point, $l<i, j<n+l, p \in E_{l}(z)$ and $q \prec p$. Then

$$
\arg \left(\overrightarrow{v_{l} v_{l-1}}\right) \leqslant \arg (\overrightarrow{p q}) \leqslant \arg \left(\overrightarrow{v_{l} v_{l+1}}\right)
$$

so $q \in E_{l}(z)$. We can argue similarly if $p$ and $q$ are on the "other side", that is, $i, j \in$ $\{n+l+1, \ldots, 2 n, 1 \ldots, l-1\}$.

Suppose now that $p$ is an $E_{i}$-boundary point, $q$ is an $E_{l}$-boundary point, $l<i<n+l$, $r$ is also a boundary point, $p, q \in E_{l}(z)$ and $q \prec r \prec p$. Then again

$$
\arg \left(\overrightarrow{v_{l} v_{l-1}}\right) \leqslant \arg (\overrightarrow{p r}) \leqslant \arg \left(\overrightarrow{v_{l} v_{l+1}}\right)
$$

therefore, $r \in E_{l}(z)$. Just like before, we can argue similarly in the case $i \in\{n+l+1$, $\ldots, 2 n, 1 \ldots, l-1\}$.

Finally, suppose that $p, q$, and $r$ are $E_{l}$-boundary points, $p, r \in E_{l}(z)$ and $r \prec q \prec p$. Again, it is easy to check that $r \in E_{l}(z)$. The same holds if $p, q$, and $r$ are $E_{n+l}$-boundary points.

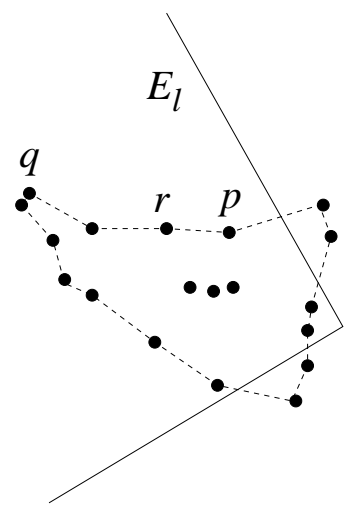

Figure 4: $E_{l}$ intersects $\mathbb{B}$ in at most two intervals

It follows from these observations that $E_{l}(z)$ intersects $\mathbb{B}$ in at most two intervals. See Figure 4. 


\subsection{Coloring algorithm}

Two boundary points are neighbors if there is no other boundary point between them. More precisely:

Definition 15. Two boundary points $p, q \in \mathbb{B}^{\prime}$ are neighbors if $p \prec q$, and either (i) there is no $r$ for which $p \prec r \prec q$, or (ii) there is no $r$ for which $r \prec p$ or $q \prec r$.

Two boundary points $p, q \in \mathbb{B}$ are neighbors if the corresponding points in $\mathbb{B}^{\prime}$ are neighbors. Let $p \sim q$ denote that $p$ and $q$ are neighbors.

Let $\approx$ be the transitive closure of the relation $\sim$ on $\mathbb{B}$, that is, $p \approx q$ if and only if there is a finite sequence of boundary points, starting with $p$, ending with $q$, such that the consecutive pairs are neighbors. The relation $\approx$ is an equivalence relation. Those boundary points $p$ which belong to an equivalence class of size one, are called lonely boundary points. The others, which have a neighbor, are called social boundary points.

As an illustration, suppose that none of the sides of $S$ are horizontal, and let $\mathcal{H}$ be set of points $(x, 0)$ where $0 \leqslant x \leqslant 10$ and $x$ is an integer. Then all points of $\mathcal{H}$ are social boundary points and all belong to same equivalence class. Now let $\mathcal{H}$ be set of points $(x, 0)$ where $0 \leqslant x \leqslant 10$ and $x$ is rational. Then all points of $\mathcal{H}$ are lonely boundary points.

First we give a (non-deterministic) coloring procedure which colors the points black and white. Then we apply it several times to obtain our red-blue coloring.

\section{Black-White-Boundary-Coloring $(S, \mathcal{H})$}

Divide the boundary of $\mathcal{H}, \mathbb{B}$, into equivalence classes by relation $\approx$. First we color the social boundary points.

Let $C$ be an arbitrary equivalence class, $|C|>1$. Take a maximal subset $M$ of the singular boundary points of $C$ with the property that no two of them are neighbors. Color them black. Now take a maximal subset $M^{\prime}$ of the uncolored points of $C$ with the propery that no two points of $M \cup M^{\prime}$ are neighbors. Color the points of $M^{\prime}$ black, too. Color the remaining points of $C$ white. It is not hard to check, that no two consecutive boundary points are black and no three consecutive are white. Do the same for each equivalence class $|C|>1$.

Now we color the lonely boundary points, denote their set by $\mathbb{B}_{\text {lonely. }}$. It is the union of at most $2 n$ homogeneous intervals, that is, $\mathbb{B}_{\text {lonely }}=\cup_{i=1}^{2 n} I_{i}$ where the elements of $I_{i}$ are all $E_{i}$-boundary points. We color each interval separately. Recall that, based on projection $\pi_{i}$, we defined the midpoint, the first and the second half of a homogeneous interval.

For each $i$, consider interval $I_{i}$. If it contains infinitely many points, color one of them black. Then again, for each $i$, if $I_{i}$ contains infinitely many points, color an uncolored one white. If it contains finitely many points, do not color them yet. Now cut each interval, which contained infinitely many points, in half, and drop (that is, do not consider anymore) intervals with finitely many points. Let $J_{1}, J_{2}, \ldots, J_{m}$ 
be the set of new intervals. Repeat the previous step, choose an uncolored point in each of the intervals with infinitely many points, and color them black, then the same with white. Cut each interval, which contained infinitely many points, in half, and drop intervals with finitely many points. Repeat this countably infinitely many times.

Finally, color all uncolored points white.

Claim 16. If there are infinitely many points in an interval of $\mathbb{B}$, then it contains infinitely many points of both colors.

Proof. We can assume that interval $I$ is homogeneous, say, all of its points are $E_{i}$-boundary points. Suppose first that $I$ contains a lonely boundary point $p$ in its interior. Then there is an accumulation point (with respect to the usual Euclidean metric), $q$, of boundary points in the interior of $I$. (Note that $q$ is not necessarily an element of $\mathcal{H}$.) If $q$ is an accumulation point of lonely boundary points, then our procedure BLACK-WHITEBoundary-Coloring $(S, \mathcal{H})$ will arrive to an interval $J \subset I$ which contains infinitely many lonely boundary points, and it colors one of them white, one black. Moreover, the procedure will find such intervals in infinitely many steps, so it colors infinitely many points white, and infinitely many black.

If $q$ is an accumulation point of social boundary points, or if $I$ does not contain a lonely boundary point $p$ in its interior, then $I$ contains infinitely many social boundary points. Then either $I$ contains three consecutive such points, or contains two consecutive that form an equivalence class of size two. In both cases, at least one of them is white and at least one is black. We can proceed similarly to find infinitely many points of both colors.

Definition 17. A boundary point $p$ is called rich if there is a translate of an $S$-wedge $E_{i}$, such that $p$ is the only boundary point in it, but it contains at least one interior point, see Figure 5.

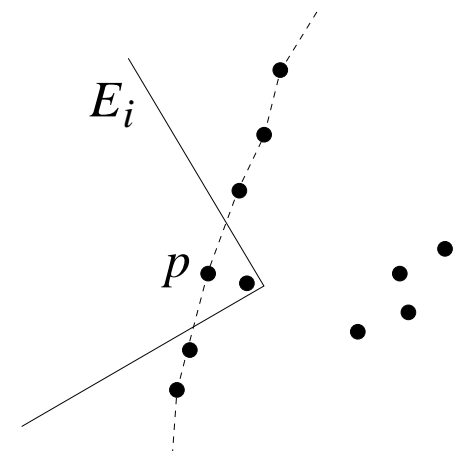

Figure 5: Point $p$ is a rich boundary point 


\subsection{Proof of Theorem 6}

Now we are ready to prove Theorem 6 . Suppose that $S$ is a closed, centrally symmetric convex polygon, its vertices are $v_{1}, v_{2}, \ldots, v_{2 n}$, ordered clockwise, the $S$-wedges are $E_{1}$, $E_{2}, \ldots, E_{2 n}$. Let $S^{\prime}$ be $S$ minus its boundary. Let $\mathcal{H}$ be a bounded set of points.

First we color the boundary, $\mathbb{B}$, of $\mathcal{H}$, then we color the boundary $\mathbb{B}^{(1)}$ of the interior points, and finally we color the remaining points. Very roughly speaking, the first level will be "responsible" for color blue in wedges which contain many, but finitely many points, the next level is responsible for color red, and coloring of the remaining points settles the wedges with infinitely many points.

\section{Red-Blue-Coloring $(S, \mathcal{H})$}

1. Let $\mathbb{B}$ be the boundary of $\mathcal{H}$ with respect to $S$. Color $\mathbb{B}$, the first level, with procedure Black-White-Boundary-Coloring $(S, \mathcal{H})$. Then, let $p \in \mathbb{B}$ be

- blue, if it is rich or colored white,

- red otherwise.

Now let $\mathcal{H}^{(1)}=\mathcal{H} \backslash \mathbb{B}$ be the set of interior points.

2. Let $\mathbb{B}^{(1)}$ be the boundary of $\mathcal{H}^{(1)}$ with respect to $S$. Color all points of $\mathbb{B}^{(1)}$, the second level, red. Let $\mathcal{H}^{(2)}=\mathcal{H}^{(1)} \backslash \mathbb{B}^{(1)}$, the set of interior points of $\mathcal{H}^{(1)}$.

3. Let $\mathbb{B}^{(2)}$ be the third level, boundary of $\mathcal{H}^{(2)}$ with respect to $S^{\prime}$. (Watch out, $S^{\prime}$ and not $S$ !) Color the points of $\mathbb{B}^{(2)}$ with procedure Black-White-Boundary$\operatorname{Coloring}\left(S^{\prime}, \mathcal{H}^{(2)}\right)$. Then, let $p \in \mathbb{B}^{(2)}$ be

- blue, if it is colored white,

- red if it is colored black.

Finally, let $\mathcal{H}^{(3)}=\mathcal{H}^{(2)} \backslash \mathbb{B}^{(2)}$, the fourth level, set of interior points of $\mathcal{H}^{(2)}$, the set of still uncolored points.

4. Take a square that contains $\mathcal{H}^{(2)}$. If it contains finitely many points of $\mathcal{H}^{(2)}$ (that is, $\mathcal{H}^{(2)}$ has finitely many points), color them red and stop. If it contains infinitely many points of $\mathcal{H}^{(2)}$, then color one red and one blue. Divide the square into four smaller squares. In each of them, if there are finitely many points of $\mathcal{H}^{(2)}$, then color all uncolored points red, and do not consider this square anymore. If there are infinitely many points in it, then color an uncolored point red and another uncolored one blue. Then divide it into four little squares. (Let common vertical boundaries belong to the left squares, common horizontal boundaries belong to the lower squares.) Repeat this infinitely many times. Finally color all points, which are still uncolored, red. 
Now we prove that this coloring satisfies the conditions. Suppose that $E_{i}(a)$ contains finitely many points of $\mathcal{H}$, but at least 9 . Then $E_{i}(a)$ contains at least one boundary point of $\mathcal{H}$.

Case 1. $E_{i}(a)$ contains one point from the first level, that is, $\left|E_{i}(a) \cap \mathbb{B}\right|=1$. Then this point is rich, so it is blue, and $E_{i}(a)$ contains at least 8 interior points.

Case 2. $\left|E_{i}(a) \cap \mathbb{B}\right|=2$. By Claim $14, E_{i}(a)$ intersects $\mathbb{B}$ in one or two intervals. In the first case $E_{i}(a)$ contains two consecutive boundary points, so one of them is blue, and it contains at least 7 interior points. In the second case both intervals contain one point, so at least one of them is rich, therefore, it is blue, and $E_{i}(a)$ contains at least 7 interior points.

Case 3. $3 \leqslant\left|E_{i}(a) \cap \mathbb{B}\right| \leqslant 8$. Since $E_{i}(a)$ intersects $\mathbb{B}$ in at most two intervals, it contains two consecutive boundary points, so one of them is blue, and $E_{i}(a)$ contains at least one interior point.

Case 4. $\left|E_{i}(a) \cap \mathbb{B}\right| \geqslant 9$. Then $E_{i}(a)$ contains at least 5 consecutive boundary points, say, $p_{1}, p_{2}, \ldots, p_{5}$. At least two of them are blue. Suppose that all of them are blue. Procedure Black-White-Boundary-Coloring $(S, \mathcal{H})$ did not color three consecutive points white, therefore, at least one of $p_{2}, p_{3}$ and $p_{4}$ got color blue, because it is rich. It is not hard to see that $E_{i}(a)$ contains the interior points corresponding to this rich boundary point.

Summarizing, if $E_{i}(a)$ contains at least 9 but finitely many points of $\mathcal{H}$, then either it contains points of both colors, or it contains a blue point on the boundary, and at least one interior point. But in this case it contains a point of $\mathbb{B}^{(1)}$, the boundary of the interior points, which is red, so we are done in this case.

Now suppose that $E_{i}(a)$ contains infinitely many points of $\mathcal{H}$, and suppose for contradiction that it does not contain points of both colors.

Case 1. $E_{i}(a)$ contains infinitely many points from the boundary of $\mathcal{H}$. By Claim $14, E_{i}(a) \cap \mathbb{B}$ consists of at most two intervals, one of the intervals, say $I$, is infinite. Procedure Black-White-Boundary-Coloring $(S, \mathcal{H})$ colors infinitely many points of $I$ to both colors. It follows immediately, that there are infinitely many blue points in $E_{i}(a)$. Therefore, by our assumption, all points of $I$ got color blue. Then infinitely many of them are rich. But then the infinitely many interior points that correspond to these rich points, are also in $E_{i}(a)$.

Case 2. $E_{i}(a)$ contains finitely many points from the boundary of $\mathcal{H}$, but at least one. Then, just like in the finite case, it is not hard to see that $E_{i}(a)$ contains at least one blue point from the boundary, and infinitely many interior points.

Case 3. $E_{i}(a)$ does not contain boundary points. Obviously, it contains infinitely many interior points.

So we can conclude that if $E_{i}(a)$ contains infinitely many points of $\mathcal{H}$, than it contains infinitely many interior points, and either it contains a blue boundary point, or no boundary points at all. Since we colored the boundary of the interior points, $\mathbb{B}^{(1)}$, red and we assumed that $E_{i}(a)$ does not contain points of both colors, either $E_{i}(a) \cap \mathbb{B}=\emptyset$, or 
$E_{i}(a) \cap \mathbb{B}^{(1)}=\emptyset$. By the definition of the boundary, if $E_{i}(a) \cap \mathbb{B}=\emptyset$, then $E_{i}(a) \cap \mathbb{B}^{(1)}=\emptyset$ as well. It follows, that $E_{i}(a) \cap \mathbb{B}^{(1)}=\emptyset$, therefore, $E_{i}(a)$ contains infinitely many points from $\mathcal{H}^{(2)}$, the set of interior points of $\mathcal{H}^{(1)}$. We distinguish two cases.

Case 1. $E_{i}(a)$ contains infinitely many points from $\mathbb{B}^{(2)}$, the boundary of $\mathcal{H}^{(2)}$ with respect to $S^{\prime}$. The set $E_{i}(a) \cap \mathbb{B}^{(2)}$ is again the union of at most two intervals, therefore, one of them contain infinitely many points, so by Claim 16 it contains infinitely many points of both colors.

Case 2. $E_{i}(a)$ contains finitely many points from $\mathbb{B}^{(2)}$. Then it contains infinitely many points from the set $\mathcal{H}^{(3)}$, the interior points of $\mathcal{H}^{(2)}$, with respect to $S^{\prime}$. We claim that in this case $E_{i}(a)$ contains a point in its interior. Suppose not. Then all points in $E_{i}(a) \cap \mathcal{H}^{(2)}$ are on the boundary of $E_{i}(a)$, so they all belong to $\mathbb{B}^{(2)}$, a contradiction. Therefore, there is a point $a_{0} \in \mathcal{H}$ in the interior of $E_{i}(a)$. Clearly, $E_{i}\left(a_{0}\right)$ is also in the interior of $E_{i}(a) . E_{i}(a) \cap \mathbb{B}=\emptyset$, hence $a_{0}$ is not a boundary point of $\mathcal{H}$, so there is a point $a_{1}$ in $E_{i}\left(a_{0}\right)$. Since $a_{1}$ is not a boundary point either, there is an $a_{2}$ in $E_{i}\left(a_{1}\right)$. This way we get an infinite sequence $a_{0}, a_{1}, \ldots$ of points in $E_{i}\left(a_{0}\right)$. With the exception of finitely many, they belong to $\mathcal{H}^{(3)}$. They have an accumulation point $x$. The point $x \in E_{i}\left(a_{0}\right)$ since $E_{i}\left(a_{0}\right)$ is closed, so $x$ is in the interior of $E_{i}(a)$. Therefore, when we colored $\mathcal{H}^{(3)}$, in step 4 of procedure Red-Blue-Coloring $(S, \mathcal{H})$, once we arrived to a little square which is in $E_{i}(a)$, contains $x$, and contains infinitely many points. So we colored one of them blue and one of them red. This concludes the proof of Theorem 6

\section{Infinite-fold coverings; Proof of Theorem 3}

Just like in the proof of Theorem 2, we can take the dual of the problem, and divide the plane into small squares. Therefore, it is enough to prove the following result.

Theorem 18. Let $S$ be a closed, convex, centrally symmetric polygon, its vertices are $v_{1}$, $v_{2}, \ldots, v_{2 n}$, oriented clockwise. Then any bounded point set $\mathcal{H}$ can be colored with red and blue such that for any translate of an $S$-wedge $E_{i}(p)$, if $\left|E_{i}(p) \cap \mathcal{H}\right|=\infty$, then $E_{i}(p) \cap \mathcal{H}$ contains infinitely many red and infinitely many blue points.

Let $S^{\prime}$ be $S$ minus its boundary and for $1 \leqslant i \leqslant 2 n$, let $E_{i}^{\prime}$ be $E_{i}$ minus its boundary. That is, $E_{1}^{\prime}, E_{2}^{\prime}, \ldots, E_{2 n}^{\prime}$ are the $S^{\prime}$-wedges.

From now on, boundary of a point set is understood according to $S^{\prime}$, and not $S$. (As an illustration, if our poit set is a closed segment parallel to a side of $S$, then it has two boundary points with respect to $S$, but all of the points are boundary points with respect to $S^{\prime}$.)

- Let $\mathbb{B}=\mathbb{B}^{(0)}$ be the set of boundary points of $\mathcal{H}$. Its interior points $\mathcal{H}^{(1)}=\mathcal{H} \backslash \mathbb{B}^{(0)}$.

- Let $\mathbb{B}^{(1)}$ be the set of boundary points of $\mathcal{H}^{(1)}$. Its interior points $\mathcal{H}^{(2)}=\mathcal{H}^{(1)} \backslash \mathbb{B}^{(1)}$.

- ...

- Let $\mathbb{B}^{(n)}$ be the set of boundary points of $\mathcal{H}^{(n)}$. Its interior points $\mathcal{H}^{(n+1)}=\mathcal{H}^{(n)} \backslash \mathbb{B}^{(n)}$. 
Moreover, let $\mathbb{B}^{*}=\bigcup_{n \in \mathbb{N}} \mathbb{B}^{(n)}$, and $\mathcal{H}^{*}=\mathcal{H} \backslash \mathbb{B}^{*}$.

We call $\mathbb{B}^{(n)}$ the $n$-th boundary level of $\mathcal{H}$. Let $\mathbb{B}_{i}^{(n)}$ be the set of $E_{i}^{\prime}$-boundary points of $\mathbb{B}^{(n)}$, and let $\mathbb{B}_{i}^{*}=\bigcup_{n \in \mathbb{N}} \mathbb{B}_{i}^{(n)}$. For each $p \in \mathbb{B}^{*}$ let $h(p)=n$ if and only if $p \in \mathbb{B}^{(n)}$. That is, each $p$ is on the $h(p)$-th level. Now we are ready to give the coloring algorithm.

\section{Multiple-Red-Blue-Coloring $(S, \mathcal{H})$}

Step 1. We color a subset of $\mathbb{B}^{*}$ so that we color at most one point from each four consecutive levels. Take a square $Q_{1}$ which contains $\mathbb{B}^{*}$. Divide it into four little squares, these are $Q_{2}, Q_{3}, Q_{4}$ and $Q_{5}$. Then divide $Q_{2}$ into four little squares, these are $Q_{6}, Q_{7}, Q_{8}, Q_{9}$. Similarly, divide $Q_{3}$ to get $Q_{10}, \ldots, Q_{13}$, and continue similarly. Eventually we divide each square in the list into four little squares, and put them in the list. This way we obtain an infinite list $Q_{1}, Q_{2}, \ldots$ of squares.

In Step 1.1, if $Q_{1}$ contains infinitely many points of $\mathbb{B}^{*}$, then color one of them, $p_{1}$, red. Otherwise, we stop. In Step 1.2, if $Q_{1}$ contains infinitely many points of $\mathbb{B}^{*} \backslash \bigcup_{l<h\left(p_{1}\right)+3} \mathbb{B}^{(l)}$, then color one of them, $p_{2}$, blue. Otherwise, we stop.

In general, in Step 1. $(2 k-1)$, if $Q_{k}$ contains infinitely many points of the set $\mathbb{B}^{*} \backslash \bigcup_{l<h\left(p_{2 k-1}\right)+3} \mathbb{B}^{(l)}$, then color one of them, $p_{2 k-1}$, red. Otherwise, we stop. Then, in Step $1.2 k$, if $Q_{k}$ contains infinitely many points of the set $\mathbb{B}^{*} \backslash \bigcup_{l<h\left(p_{2 k-2}\right)+3} \mathbb{B}^{(l)}$, then color one of them, $p_{2 k}$, blue. Otherwise, we stop.

After countably infinite many steps, we are done with Step 1.

In the following steps we color the uncolored points.

Step 2. For each even $n$, color $\mathbb{B}^{(n)}$ with procedure Black-White-BoundaryColoring $\left(S^{\prime}, \mathcal{H}^{(n)}\right)$. Now a boundary point $p \in \mathbb{B}^{(n)}$ will be

- blue, if it is rich or colored white,

- red otherwise.

Step 3. For each odd $n$, color $\mathbb{B}^{(n)}$ with procedure Black-White-BoundaRY$\operatorname{Coloring}\left(S^{\prime}, \mathcal{H}^{(n)}\right)$. Now a boundary point $p \in \mathbb{B}^{(n)}$ will be

- red, if it is rich or colored white,

- blue otherwise.

That is, we change the roles of the colors. 
Step 4. Take a square which contains $\mathcal{H}^{*}$. If it contains infinitely many points from $\mathcal{H}^{*}$, (that is, $\mathcal{H}^{*}$ has infinitely many points) then color one of them blue and one of them red. Divide the square into four little squares. In each of them, which contains infinitely many points from $\mathcal{H}^{*}$, color one of the uncolored points blue and one of them red, and divide it into four smaller squares. (Again, let common vertical boundaries belong to the left squares, common horizontal boundaries belong to the lower squares.) Continue recursively. Once we obtain a square which contains only finitely many points from $\mathcal{H}^{*}$, color all uncolored points red, and do not divide it into smaller squares.

Step 5. Color all uncolored points red.

Suppose that $\mathcal{H}$ is colored by procedure Multiple-Red-Blue-Coloring $(S, \mathcal{H})$. We show that if a translate of an $S$-wedge contains infinitely many points of $\mathcal{H}$, then it contains infinitely many points of both colors. First we show that a wedge contains an accumulation point in its interior, then it contains infinitely many points of both colors.

Lemma 19. Suppose that $E_{i}(a) \cap \mathcal{H}$ is infinite and this set has an accumulation point $t$ in the interior of $E_{i}(a)$. Then $E_{i}(a)$ contains infinitely many points of both colors.

Proof. We have several cases according to the types of points that converge to $t$.

Case 1. Point $t$ is the accumulation point of the interior points $\left(\mathcal{H}^{*}\right)$. In this case, in Step 4 , we found infinitely many little squares that contain infinitely many points of $\mathcal{H}^{*}$ but contained in $E_{i}(a)$. Therefore, $E_{i}(a)$ contains infinitely many points of both colors.

Case 2. There are infinitely many boundary levels whose points converge to $t$. In this case we can argue similarly as in the previous case. In Step 1 of the procedure we produce a red and a blue sequence of points that converge to $t$. $E_{i}(a)$ contains infinitely many points of both sequences.

Case 3. Suppose now that there are only finitely many boundary levels whose points converge to $t$, and $t$ is not the accumulation point of the interior points $\left(\mathcal{H}^{*}\right)$. Let $n$ be the largest number with the property that $t$ is an accumulation point of $\mathbb{B}^{(n)}$.

Then it follows from Claim 16 that $E_{i}(a)$ contains infinitely many black and white points. If $E_{i}(a)$ does not contain infinitely many red and blue points, then there is a sequence $p_{1}, p_{2}, \ldots$ of rich boundary points that converge to $t$. For each rich boundary point $p_{j} \in \mathbb{B}^{(n)}$, there is a point $p_{j}^{\prime} \in \mathcal{H}^{(n+1)}$ which "proves its richness", that is, there is a translate $E_{i}^{(j)}$ of $E_{i}$ which contains $p_{j}$ and no other boundary point of $\mathcal{H}^{(n)}$, and also contains $p_{j}^{\prime}$ of $\mathcal{H}^{(n+1)}$. Since the sequence $p_{1}, p_{2}, \ldots$ converges to $t$, the distance between $p_{j}$ and $p_{j+1}$ also goes to 0 as $j$ goes to infinity. Therefore, the distance between $p_{j}$ and $p_{j}^{\prime}$ also goes to 0 , so the sequence $p_{1}^{\prime}, p_{2}^{\prime}, \ldots$ converges to $t$ as well. But this contradicts our assumptions.

So, we are done if the points in $E_{i}(a)$ have an accumulation point in the interior of it. Suppose now that there is no such accumulation point. 
I. Assume that $E_{i}(a) \cap \mathbb{B}_{i}^{*}$ is infinite. Observe that if $E_{i}(a) \cap \mathbb{B}_{i}^{(n)} \neq \emptyset$, then, by the definition of the boundary levels, for every $k<n, E_{i}(a) \cap \mathbb{B}_{i}^{(k)} \neq \emptyset$.

We distinguish two subcases.

(a) Suppose that for every $n, E_{i}(a) \cap \mathbb{B}_{i}^{(n)} \neq \emptyset$. Then, since we changed the roles of the colors for the even and odd numbered levels, for $n$ even, the sets $E_{i}(a) \cap \mathbb{B}_{i}^{(n)}$ contain infinitely many blue points, for $n$ odd, they contain infinitely many red points.

(b) Suppose now, that $E_{i}(a) \cap \mathbb{B}_{i}^{(n)} \neq \emptyset$ holds only for finitely many levels. Let $n$ be the largest number such that $E_{i}(a) \cap \mathbb{B}^{(n)}$ is infinite. By Claim 16, procedure BLACK-White$\operatorname{BOUNDARY-COLORING}\left(S, \mathcal{H}^{(n)}\right)$ colors infinitely many points of $E_{i}(a) \cap \mathbb{B}^{(n)}$ black and white. So, the only problem could be, that infinitely many black points among these are rich. Now we can argue similarly as in Case 3 . Let $p_{1}, p_{2}, \ldots$ be a sequence of of rich boundary points in $\mathbb{B}^{(n)}$. For each $p_{j} \in \mathbb{B}^{(n)}$, there is a point $p_{j}^{\prime} \in \mathcal{H}^{(n+1)}$, and a translate of $E_{i}^{(j)}$ of $E_{i}$ which "proves its richness". But then $E_{i}(a)$ also contains the sequence $p_{1}^{\prime}, p_{2}^{\prime}, \ldots$. Since $n$ is the largest number such that $E_{i}(a) \cap \mathbb{B}^{(n)}$ is infinite, only finitely many of $p_{1}^{\prime}, p_{2}^{\prime}, \ldots$ could belong to $\mathbb{B}_{i}^{*}$. On the other hand, if any $E_{i}^{(j)}$ contains infinitely many points of $\mathcal{H}^{(n+1)}$, then they have an accumulation point which is in the interior of $E_{i}(a)$, contradicting our assumption. Therefore, each $E_{i}^{(j)}$ contains only finitely many points of $\mathcal{H}^{(n+1)}$. But then they all belong to some boundary level greater than $n$. It is a contradiction again, since we assumed that there are only finitely boundary levels greater than $n$ which contain a point in $E_{i}(a)$ and each contain only finitely many.

II. Finally, suppose that $E_{i}(a) \cap \mathbb{B}_{i}^{*}$ is finite. We can assume without loss of generality that it is empty. We assumed that there is no accumulation point in the interior of $E_{i}(a)$. If $E_{i}(a)$ does not contain any point in its interior, we have a contradiction, since in this case all points in $E_{i}(a)$ are $E_{i}$-boundary points. If it contains a point $p_{0}$ in its interior, then, since it is not an $E_{i}$-boundary point, $E_{i}\left(p_{0}\right)$ contains a point $p_{1}$. Since it is not an $E_{i}$-boundary point either, $E_{i}\left(p_{1}\right)$ also contains a point $p_{2}$. We get an infinite sequence $p_{1}, p_{2}, \ldots$ in $E_{i}\left(p_{0}\right)$, so they have an accumulation point $t$ in the interior of $E_{i}(a)$. It is again a contradiction.

This concludes the proof of Theorem 18, and therefore we also proved Theorem 3.

\section{Remarks; Other versions of cover-decomposability}

1. The concept of cover-decomposability has many other versions, instead of the plane, we can consider multiple coverings of an arbitrary set, we can assume that we have finitely many, countably many, or arbitrary many translates in the covering. These versions are sometimes confused in the literature, moreover, there are some incorrect statements because not the correct version of cover-decomposability is used. See [8] and [6] for an overview.

Every covering in the sequel is a family of translates of a planar set $S$.

Definition 20. (a) A covering is finite, if it contains finitely many translates.

(b) A covering is locally finite, if any compact set intersects only finitely many translates. 
(c) A covering is countable, if it contains countably many translates.

Now we define eight different versions of cover-decomposability.

Definition 21. A planar set $S$ is

\{finite, locally finite, countable, or arbitrary\}

\{plane- or total- $\}$

cover-decomposable, if there is a constant $k$ such that any

\{finite, locally finite, countable, or arbitrary\}

$k$-fold covering of

$\{$ the plane, or any planar set\}

can be decomposed into two coverings.

Our Theorem 2 states that every centrally symmetric closed convex polygon is planearbitrary-cover-decomposable. It is not hard to see, that our proof works also for the other versions of cover-decomposability. Cover-decomposability was known only for those versions which could be reduced to a finite problem. The next table summarizes the references for all positive results for centrally symmetric closed convex polygons. [KT] refers to the present paper.

\begin{tabular}{c|c|c|c|c|} 
& finite & locally finite & countable & arbitrary many \\
\hline the plane & - & {$[5]$} & {$[\mathrm{KT}]$} & {$[\mathrm{KT}]$} \\
\hline any planar set & {$[5]$} & {$[5]$} & {$[\mathrm{KT}]$} & {$[\mathrm{KT}]$} \\
\hline
\end{tabular}

Our proof, with very little modification, implies the same results for open centrally symmetric convex polygons. In this case it is easier to reduce the problem to the finite case, therefore, cover-decomposability was proved for more versions. The next table summarizes the situation for centrally symmetric open convex polygons.

\begin{tabular}{c|c|c|c|c|} 
& finite & locally finite & countable & arbitrary many \\
\hline the plane & - & {$[5]$} & {$[5]$} & {$[5]$} \\
\hline any planar set & {$[5]$} & {$[5]$} & {$[\mathrm{KT}]$} & {$[\mathrm{KT}]$} \\
\hline
\end{tabular}

2. In the proof of Theorems 2 and 3 we used different colorings. In fact, there is a single coloring algorithm which can be used in both proofs, but we found it too technical to present it.

\section{Acknowledgements}

We are very grateful to Balázs Keszegh for his remarks and suggestions. 


\section{References}

[1] P. Brass, J. Pach, and W. Moser: Research Problems in Discrete Geometry, Springer, Berlin, 2005.

[2] M. Elekes, T. Mátrai, and L. Soukup: On splitting infinite-fold covers, Fundamenta Mathematicae 212:95-127, 2011.

[3] M. Gibson and K. Varadarajan: Optimally decomposing coverings with translates of a convex polygon, Discrete $\&$ Computational Geometry 46:313-333, 2011. Also in: Proc. 50th Annual IEEE Symposium on Foundations of Computer Science,(FOCS 09), 159-168, IEEE Computer Soc., Los Alamitos, CA, 2009.

[4] J. Pach: Decomposition of multiple packing and covering, Diskrete Geometrie, 2. Kolloq. 169-178, Math. Inst. Univ. Salzburg, 1980.

[5] J. Pach: Covering the plane with convex polygons, Discrete $\&$ Computational Geometry 1:73-81, 1986.

[6] J. Pach, D. Pálvölgyi and G. Tóth: Survey on Decomposition of Multiple Coverings, Geometry-Intuitive, Discrete and Convex, Bolyai Math. Soc. Studies, I. Bárány et al, eds, 24:219-259, 2013.

[7] J. Pach, G. Tardos, and G. Tóth: Indecomposable coverings, Canadian Mathematical Bulletin 52:451-463, 2009. Also in: The China-Japan Joint Conference on Discrete Geometry, Combinatorics and Graph Theory (CJCDGCGT 2005), Lecture Notes in Computer Science 4381:135-148, Springer, Berlin, 2007.

[8] D. Pálvölgyi: Indecomposable coverings with concave polygons, Discrete \& Computational Geometry 44:577-588, 2010.

[9] D. Pálvölgyi: Indecomposable coverings with unit discs, manuscript, arXiv: 1310.6900

[10] D. Pálvölgyi and G. Tóth: Convex polygons are cover-decomposable, Discrete \& Computational Geometry 43:483-496, 2010.

[11] G. Tardos and G. Tóth: Multiple coverings of the plane with triangles, Discrete $\mathcal{G}$ Computational Geometry 38:443-450, 2007. 